AIAA-2001-1307

\title{
DYNAMIC MODELING AND TESTING OF MSRR-1 FOR USE IN MICROGRAVITY ENVIRONMENTS ANALYSIS
}

\author{
Christy Gattis \\ NASA Marshall Space Flight Center \\ ED21 \\ Huntsville, AL 35812 \\ christy.gattis@msfi.nasa.gov \\ Bruce LaVerde \\ ERC Incorporated \\ 555 Sparkman Drive, Executive Plaza, Suite 1622 \\ Huntsville, AL 35816 \\ bruce.laverde@msfc.nasa.gov \\ Mike Howell \\ Qualis Corporation \\ 3225 Bob Wallace Avenue, Suite C \\ Huntsville, AL 35805 \\ michael.howell@msfc.nasa.gov
}

\begin{abstract}
Delicate microgravity science is unlikely to succeed on the International Space Station if vibratory and transient disturbers corrupt the environment. An analytical approach to compute the on-orbit acceleration environment at science experiment locations within a standard payload rack resulting from these disturbers is presented. This approach has been grounded by correlation and comparison to testverified transfer functions. The method combines the results of finite element and statistical energy analysis using tested damping and modal characteristics to provide a reasonable approximation of the total root-mean-square (RMS) acceleration spectra at the interface to microgravity science experiment hardware.
\end{abstract}

\section{INTRODUCTION}

Figure 1 presents the First Materials Science Research Rack (MSRR-1), which is part of the

Copyright (C) 2001 by the American Institute of Aeronautics and Astronautics, Inc. No copyright is asserted in the United States under Title 17, U.S. Code. The U.S. Government has a royalty-free license to exercise all rights under the copyright claimed herein for Governmental purposes. All other rights are reserved by the copyright owner.
Materials Science Research Facility (MSRF). MSRF is a multi-user, multi-purpose facility for materials science research aboard the International Space Station. MSRF experiments will provide fundamental and unique scientific knowledge of phenomena affected or obscured by gravity. These investigations focus on identifying the effect of gravity on these phenomena. Therefore, the microgravity environment in which each experiment is conducted must be evaluated and compared to the requirements for microgravity science [1].

The environment on board Station is not a constant "micro-g" (order of magnitude $\sim 1 \times 10^{-6} \mathrm{~g}$ ) level. Fans, pumps, motors, and all moving parts create dynamic disturbances, which must be accounted for in the environment at the experiment location. In order to fully characterize the microgravity environment at an experiment, one must understand how vibratory disturbances throughout the Station and within the payload are transmitted through the payload rack.

A test was conducted to determine a set of transfer functions (TF), or frequency response functions (FRF), that defines the acceleration at a point of interest resulting from a unit force input at some other disturber location on the rack structure [2]. By applying inputs and retrieving responses at several "structurally diverse" locations within the rack (i.e. different types of structural 


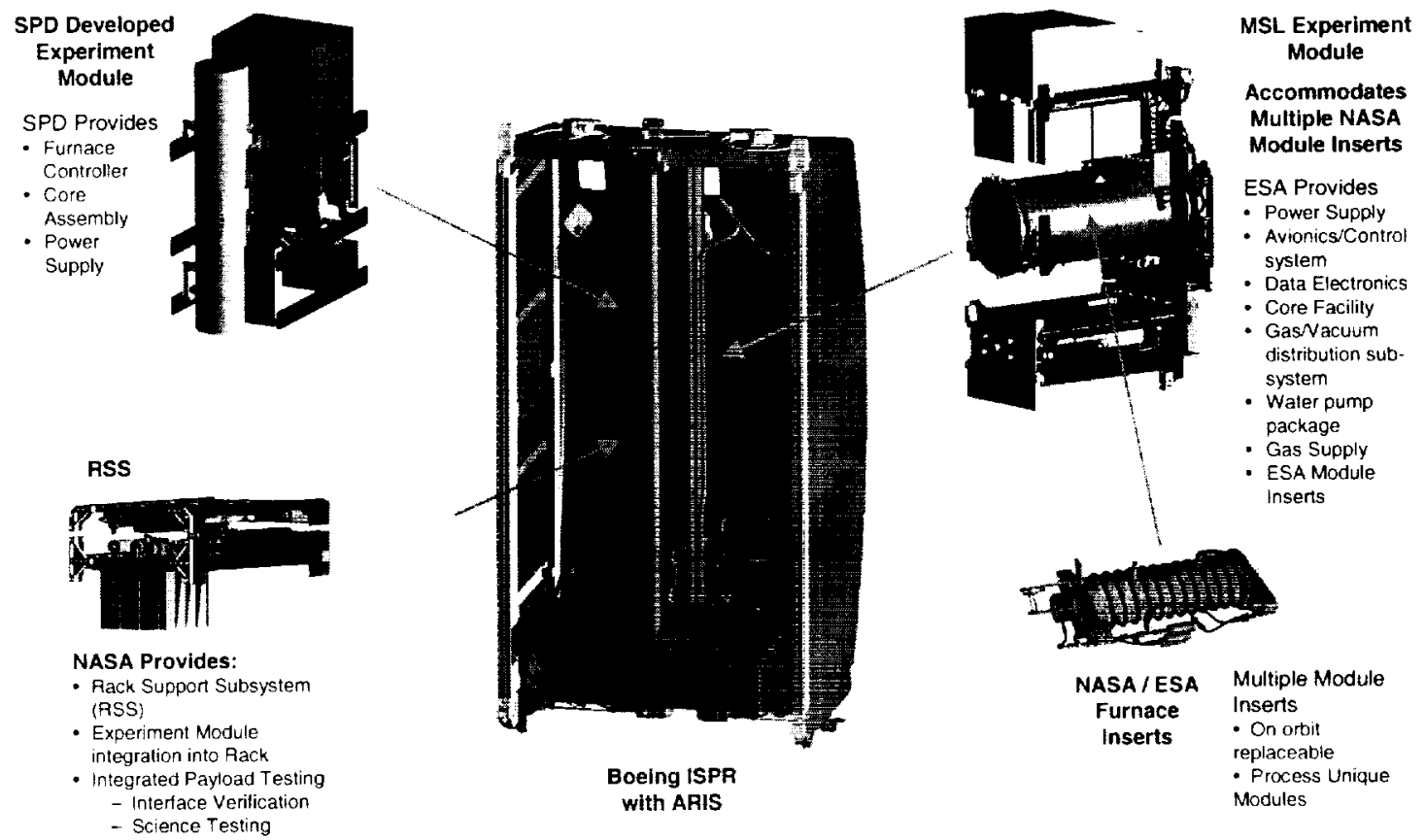

Figure 1: MSRR-1 Rack with Materials Science Research Payloads

relationships, such as post input/panel output, shelf input/post output, etc.), it was possible to generally characterize the transmission paths though the rack structure. characterize the transmission paths though the rack structure.

The response to disturbances in both the vibratory and transient regimes has been computed using a set of analytical TF. Acoustic and structural interaction has also been considered. Transfer functions from each disturber location to the science interface have been computed using both finite element (FEA) and statistical energy analysis (SEA). These analyses have also been anchored to the test results measured during transfer function testing.

\section{COMPONENT DISTURBANCE FORCES}

Characterization of disturbance forces is an important input to the microgravity analysis. Disturbance force spectra exerted by an operating component can be directly measured at the component attachment points. When measured forces are not available scaling factors have been applied to measurements from similar disturbers.

To make direct force measurements, the disturbing component must be attached to a rigid mass, i.e. something much stiffer and heavier than the component. Useful measurements have been obtained using a heavy steel plate fixture bolted to a concrete floor. The first frequency of the disturber/fixture system should be higher than the range of interest of the measurement. Triaxial force sensors at each attach location are used to record forces in the $x, y$ and $z$ axes as the equipment is operated.

Figure 2 illustrates installation of the tri-axial Kistler Model 9017A washer-type force sensors. Washers were added so that the force sensor would lie flat, allowing the connector to clear both the fixture plate and the bottom of the component. The preload bolt for each force sensor was placed through an existing hole in the component. The preload nut was placed on top of the component. This configuration allows the application of the proper preload needed for force sensor operation. Time histories were recorded and used to produce power spectra and $1 / 3$ octave band spectral measurements. Figure 3 provides an example of measured disturbance force from a fluid loop coldplate operated at a range of pressures.

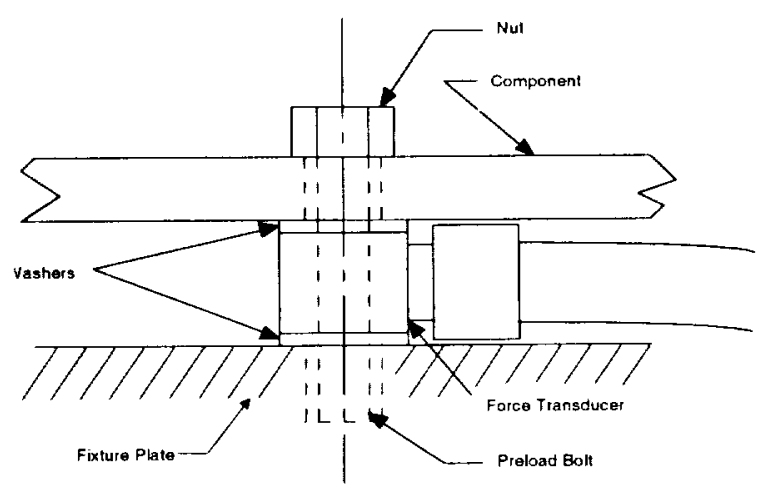

Figure 2: Disturber Force Sensor Installation for Direct Force Measurement 


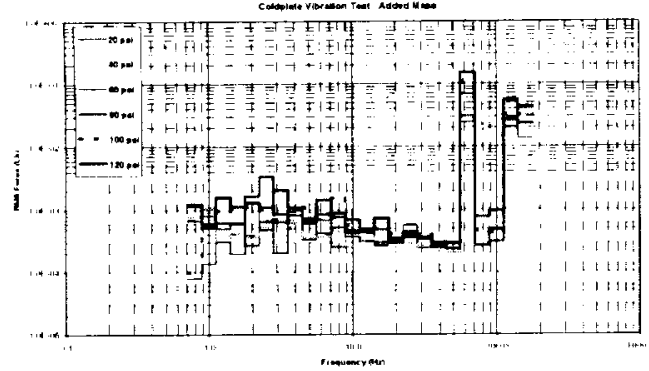

Figure 3: Direct Measurements of Coldplate Disturbance Force Spectra

\section{TRANSFER FUNCTION TEST}

The transfer function test was conducted at Marshall Space Flight Center using a flight-qualified International Standard Payload Rack (ISPR). This ISPR was configured with mass simulators to represent the MSRR-1 rack configuration. Although the weight and CG contributions of the ARIS actuator assemblies were included in the overall rack, there were no masses attached to the rack side panels to represent those actuators.

The rack was suspended in a horizontal configuration, using bungee cords at the front four corners, to simulate a free-free boundary condition (Figure 4). Rigid body modes were observed to be under $1 \mathrm{Hertz}(\mathrm{Hz})$.

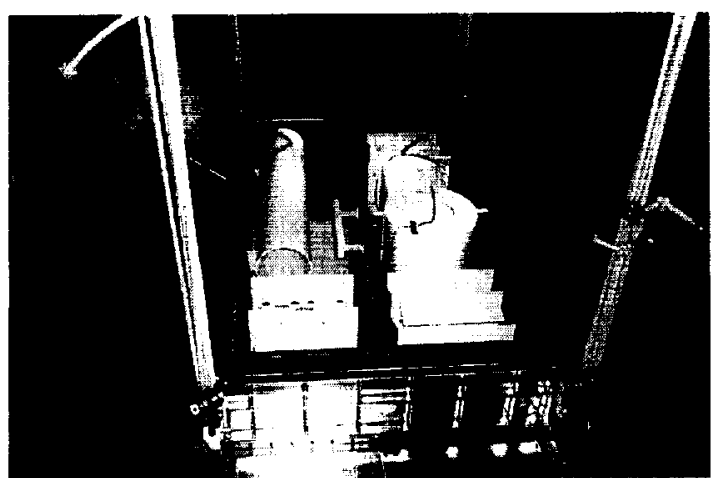

Figure 4: MSRR-1 Rack with Mass Simulators

Configured for Transfer Function Test

Excitation was applied at 9 locations in each of 3 axes independently using an impact hammer instrumented with a PCB208A03 load cell. For each impact location, frequency responses were measured at 12 locations by PCB Model 333 triaxial accelerometers. The force level of the impacts was 10 to $15 \mathrm{lbs}$ peak, with the bandwidth of interest being from 0 to $400 \mathrm{~Hz}$, based on experiment and Station requirements. For each impact, the responses were measured at each of the 12 triaxial locations and the frequency response functions (FRFs) were recorded

When taking impact data, an exponential window is applied to the response to reduce leakage during computation of the FRF. This windowing adds an "artificial" damping to the functions. Because damping values were to be obtained from these functions, the effect of the exponential window was taken into account. The exponential time constant used for these measurements was 1.33 seconds.

Modal damping ratios for primary structural modes were calculated from the TF test data using the halfpower point method [3]. These are summarized in Table 1. Damping for the first predominant structural mode at $27 \mathrm{~Hz}$ was $0.97 \%$, almost four times the conservative value, $0.25 \%$, specified for analysis when test-documented damping values are not available. The average value over the frequency range 46 to $94 \mathrm{~Hz}$ was $0.53 \%$. These tested damping parameters were applied in both the SEA and FEA analyses.

\begin{tabular}{|c|c|c|}
\hline & $\begin{array}{c}\text { Window Energy } \\
\text { Uncorrected }\end{array}$ & \begin{tabular}{l} 
Window Energy \\
Corrected \\
\hline Freg. H2.
\end{tabular} Damping, \% Crit \\
\hline 27 & 1.41 & 0.97 \\
\hline 46 & 0.65 & 0.39 \\
\hline 51 & 0.98 & 0.75 \\
\hline 84 & 0.6 & 0.45 \\
\hline 94 & 0.64 & 0.51 \\
\hline & & \\
\hline $46-94$ & Average Over This Range $=$ & 0.525 \\
\hline
\end{tabular}

Table 1: Damping Calculated From Test Data Using the Half-Power Method

\section{FINITE ELEMENT ANALYSIS}

The NASTRAN finite element model (FEM) used to simulate the transfer function test article was derived from the Boeing ISPR stress model, which had been correlated to rack qualification modal test data. The model was modified by replacing some of the solid elements with bar elements, to make it less costly for the dynamic analysis.

This initial MSRR-1 dynamic FEM was modified to match the test configuration with poor results. The modifications included removing the front faceplate and certain Active Rack Isolation System (ARIS) lumped mass hardware representations from the model to better match the test configuration. However, the FEA frequency response results did not 
correlate well with the test results at low frequencies using the existing model.

The model was therefore improved by removing the existing representations for on-orbit internal rack components. These were replaced with more detailed representations of the mass simulator hardware. A comparison of the component model representations can be seen in Figures 5 and 6 .
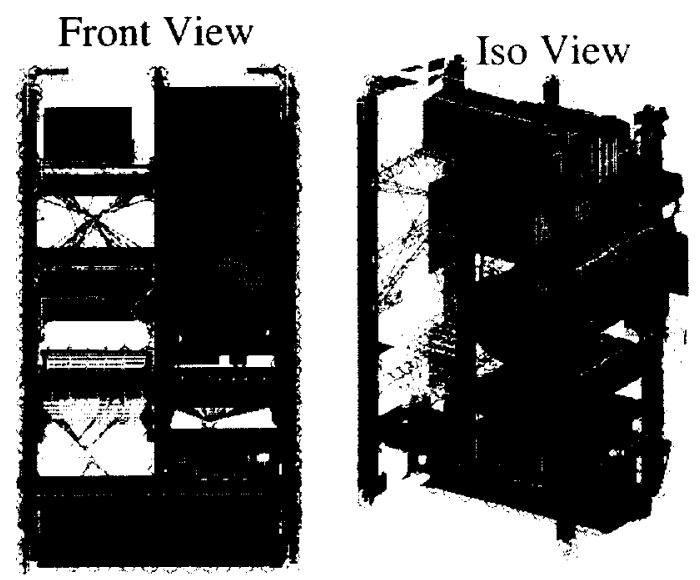

Figure 5: Component Representations from Initial MSRR-1 FEM Model
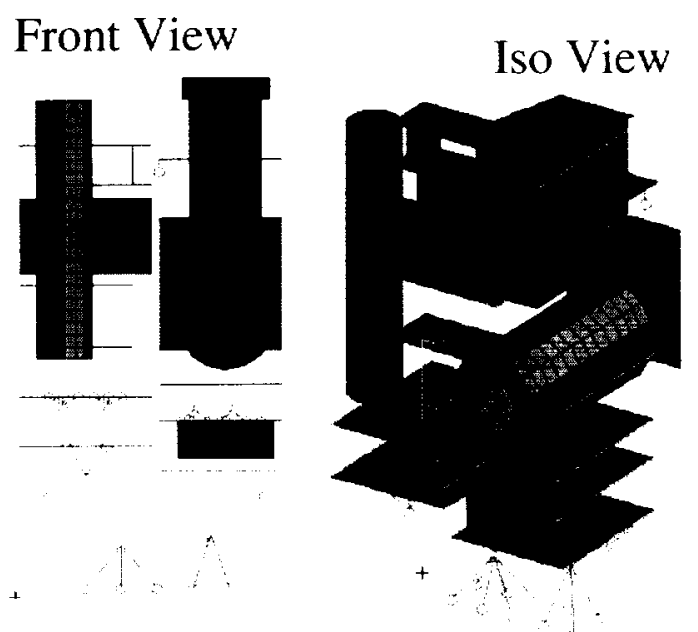

Figure 6: Revised Component Representations for MSRR-1 FEM Model

The FEA results using the new component representations were considerably improved. Figures 7 and 8 provide an example of how the finite element results compared with test before and after this final model revision respectively. Figure 8 shows that the results for the revised model match much better at the low frequencies. This is especially true for the first mode at $27 \mathrm{~Hz}$. Thus, the test results were also used to indicate the need for greater model fidelity. The revised finite element model consists of 9.897 elements $(8,376$ quadralaterals, 1,044 triangular, 358 bar, and 119 point elements) and 10,001 nodes.

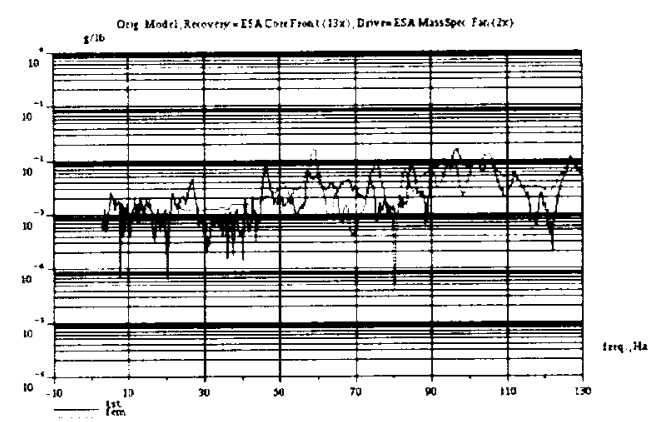

Figure 7: Measured vs Analytical TF from Initial FEM Model of MSRR-1 Rack

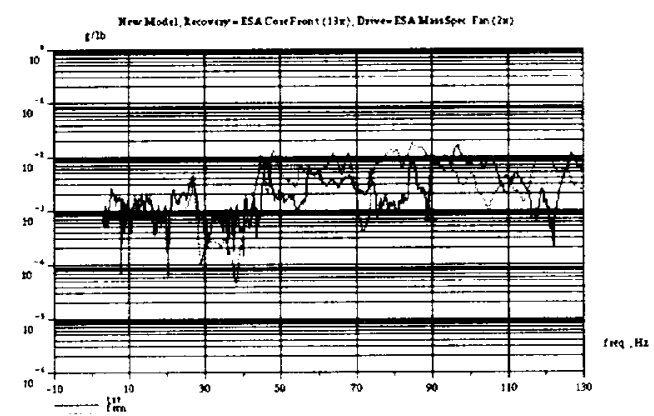

Figure 8: Measured vs Analytical TF from Revised Model of MSRR-1 Rack

A NASTRAN frequency response function analysis was performed to develop $\mathrm{TF}$ for each disturber location. The results are written to a NASTRAN punch file. These punch files are labeled according to the disturber location and direction. A Unix native programming language, which was convenient for processing text files, was run. This language extracts the responses from the punch file and performs one third octave band averaging and writes the results to files with names which indicate disturber and response location and direction. The Lanczos procedure (NASTRAN Solution 103) was used to extract the eigenvalues and eigenvectors. This information was saved in the database so that restart frequency response runs (NASTRAN Solution 111) could be performed. This method is efficient since the costly modal extraction procedure is performed only once.

\section{STATISTICAL ENERGY ANALYSIS (SEA)}

An SEA model of the MSRR-1 rack was generated using the AutoSEA 2.1 software analysis tool Figure 9. Both the structural elements and the internal 
acoustic cavities were included for vibration transfer function studies. The model was used to produce transfer functions describing the response at the rack interface to the science experiments due to unit forces applied at disturber locations within the rack. Transfer functions from acoustic disturbances in the internal acoustic cavity were also considered.

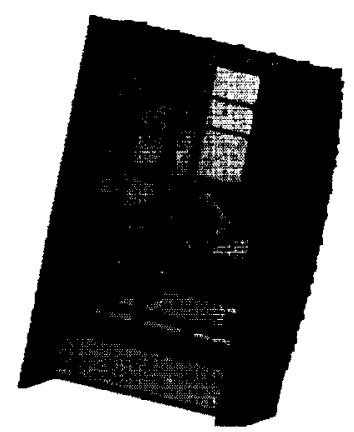

Figure 9: SEA Model of MSRR-1 without Front Faceplate

The SEA approach uses a frequency band limited energy balance to simplify the job of estimating average response of model subsystems to power inputs. SEA provides an estimate of vibration transmission based on energy flow between subsystems. The SEA solution provides a fair estimate of the average response in frequency bands where the modal density exceeds 6 modes per band; therefore, it is useful for transfer function studies in these bands.

Comparison overlay plots of SEA TF versus TF from test are presented in Figures 10 and 11 . Since the SEA results are 1/3-octave band averages, the test results plots are also averaged in 1/3-octave bands.

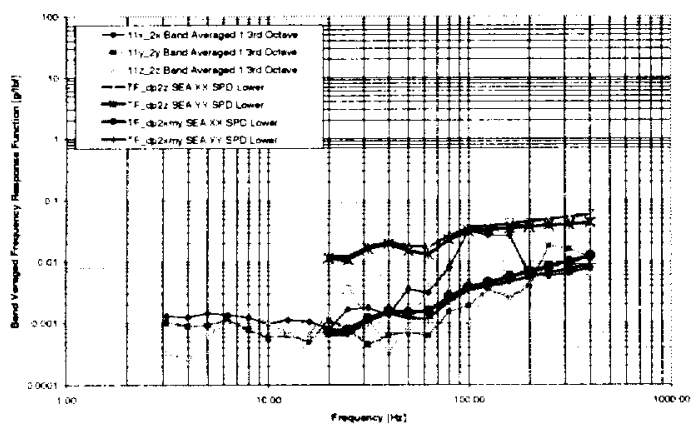

Figure 10: Typical Overlay of 1/3 Octave Band SEA $\mathrm{TF}$ to $\mathrm{TF}$ from Test at Science Interface

The comparison of SEA results to test data showed two different trends. First, when the excitation force is applied in-plane with the structural members the TF produced runs through the middle of the test data , i.e. test data falls both above and below the analytical predictions. Second, when the excitation is applied normal to the structural members the results tended to predict and envelope the magnitude of the largest test results. In other words, the results seemed to be conservative over most frequency bands and same order of magnitude with the highest magnitude frequency bands.

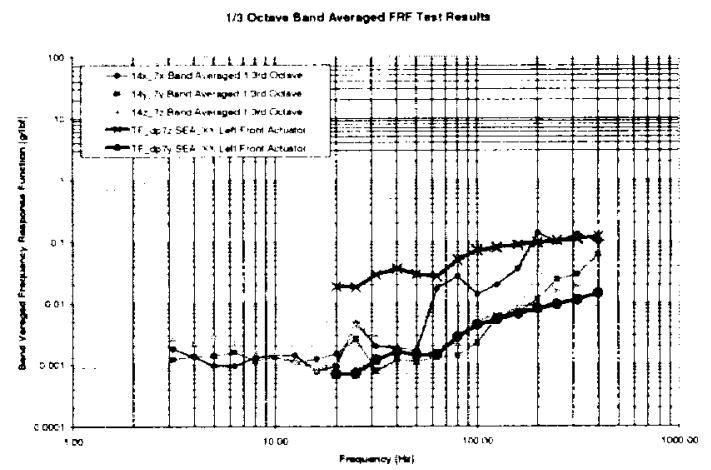

Figure 11: Overlay of 1/3 Octave Band SEA TF to TF from Test On Rack Panel

The SEA results for normal excitation may serve to keep our analyses conservative in the frequency range above $150 \mathrm{~Hz}$ where FEA results appeared low (compare Figures 12 and 13).

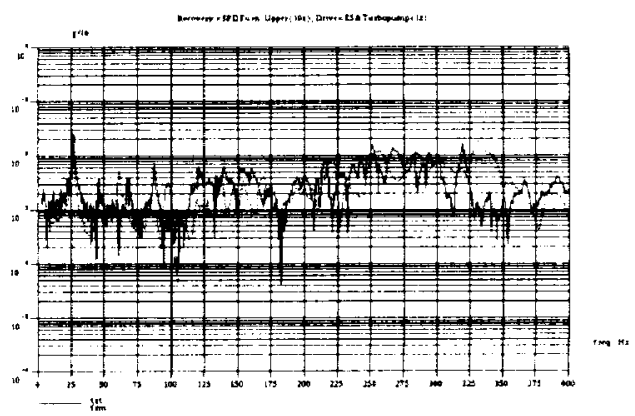

Figure 12: Energy Matching Test Across Spectrum from Finite Element Result

SEA is a powerful tool in the frequency bands where energy flow assumptions are valid. But, at lower frequencies where the number of modes per band is small and where boundary conditions have their greatest effect, FEM provides the best results. 


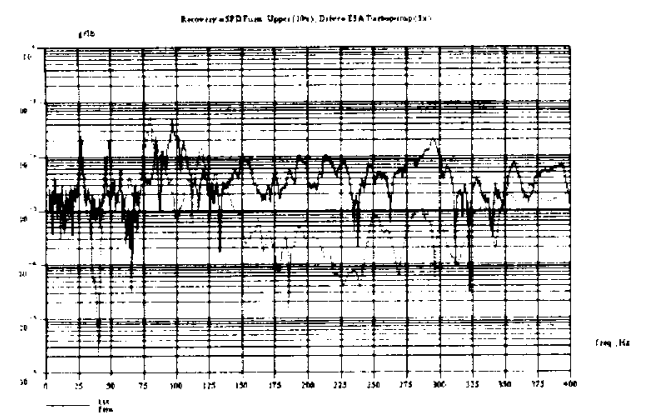

Figure 13: Energy Under Test Above $150 \mathrm{~Hz}$ Spectrum for Finite Element Result

The finite element and SEA results tended to validate each other (Figures 14-16), indicating the two could be combined, using FEA TF below $100 \mathrm{~Hz}$ and SEA above $100 \mathrm{~Hz}$. More precisely, the maximum analytical transfer function was used, whether from FEA or SEA in each $1 / 3$ octave band above $100 \mathrm{~Hz}$. This logic tended to select the SEA TF in most of the bands, except for the lowest of the SEA TFs.

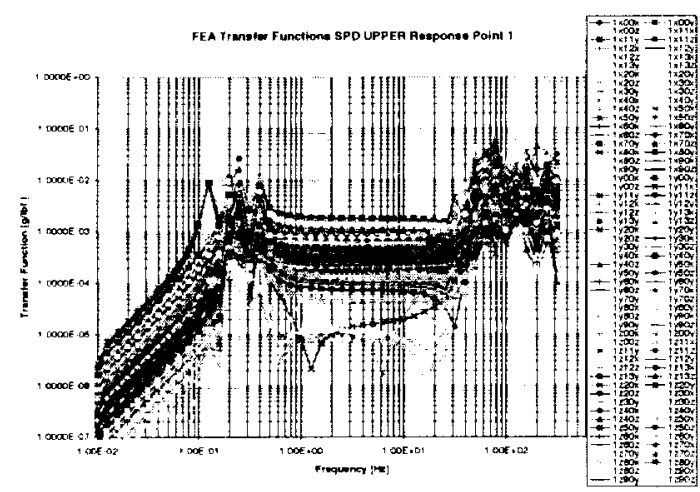

Figure 14: Typical FEM TF at Science Interface

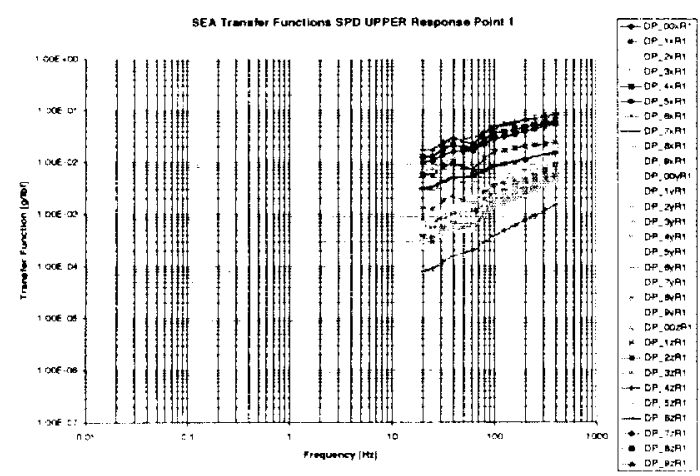

Figure 15: Typical SEA TF at Science Interface

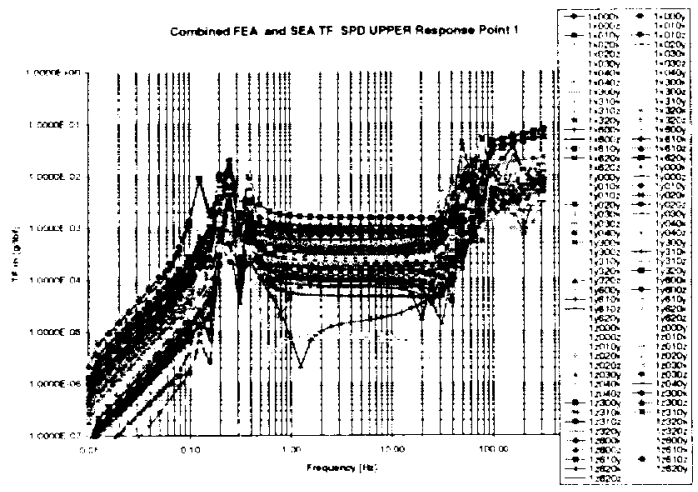

Figure 16: Combined FEM/ SEA TF Used to Calculate Acceleration Response at Science Interface

\section{RESOLVING DIFFERENCES BETWEEN TEST AND ON-ORBIT RACK CONFIGURATIONS}

It should be noted that some differences exist between the test article and the on-orbit configuration of the rack. Two types of changes to the finite element model addressed these differences. First, lumped masses were added to the model to account for the presence of ARIS hardware mounted to the top and side panels of the rack. Also, a stiffness matrix was used to represent the on-orbit attachment of the rack to the US Laboratory module.

The test-correlated finite element model was configured to match the mass and boundary conditions when installed in the orbiting Space Station. The face plates and ARIS mass and stiffness were added to the model.

ARIS attenuates vibration between the MSRR-1 rack and the Station. The net rack to Station stiffness with respect to the geometric center of the MSRR-1 rack was modeled by attaching CELAS spring elements across two coincident nodes located at geometric center of the rack. One node is grounded and the other node is connected to the model at the locations of the ARIS actuators with RBE2 elements. The DOF associated with each ARIS actuator direction are constrained to move with the ungrounded node at the geometric center. The net stiffness values were measured from tests done with the rack powered off.

The stiffness of these springs is small in comparison with the rack, which causes the frequency of the first six modes to be very small, ranging from 0.12 to 0.41 $\mathrm{Hz}$. The first three flexible body modes are 24,25 . and $34 \mathrm{~Hz}$. 


\section{RESULTS}

The analysis flow is summarized in three steps:

1. Determine best available disturbance force spectra.

2. Multiply these force spectra by the appropriate transfer functions to generate individual acceleration response spectra at the science interfaces.

3. Finally, the individual acceleration spectra at the science interface are combined using a root-sum-square approach to characterize the total acceleration environment at each science interface.

Figure 17 shows an example of the assessment of the acceleration environment at an experiment interface.

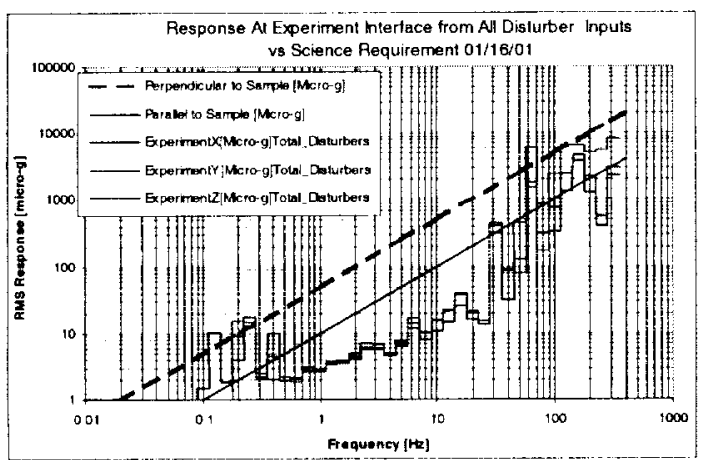

Figure 17: Example of Assessment of Acceleration Environment at Experiment Interface

Processing the disturbance and transfer functions was automated since there were many to work with. Acceleration response calculations were taken for the rack to science experiment interfaces. Each experiment furnace was assessed at two attach points, for a total of four acceleration response locations. Net rack to station interface forces and moments were recovered at the rack geometric center ground point, for a total of six response recovery locations. Eleven fan, pump, and coldplate locations were used to apply the disturbances. Each disturber force and response point has three component directions so that the total number of transfer functions is:

$$
\text { TF Total }=(11 \times 3) \times(6 \times 3)=594
$$

Disturber force spectra data was applied for all of the disturber data currently available. Disturber loads were assumed to be uncorrelated, so response was calculated for each individual disturber force and direction. Root sum square (RSS) responses for any combination of disturber forces are obtained using the software package SCILAB, which is similar to MATLAB

\section{CONCLUSIONS/RECOMMENDATIONS}

The efforts to establish this acceleration environment at the science interfaces produced results that matched the test data well. Furthermore, the total acceleration predictions are close to the desired science requirement. This integrated approach should provide a conservative representation of the rack structure since our analysis does retain a level of conservatism, in that:

1. The disturber functions have not all been determined from test. Those functions that are estimates are intended to conservatively envelope the actual disturbers especially at low frequency.

2. The disturber functions from test have been applied using the same worst-case forces in all three orthogonal directions.

Because these analytical transfer functions have been properly grounded in physical characteristics determined by test, they may be useful for a statistical study to define several conservative transfer functions for use by the microgravity community.

Since experimentally determined damping loss factors can vary significantly between subsystems. Future work that would add valuable information would be a test using reverberant test times to determine subsystem and frequency band dependent damping. This data would provide a better estimate of damping in frequency bands where modal overlap makes distinguishing one mode from the next difficult.

\section{REFERENCES}

1. Microgravity Control Plan (SSP 50036B), Revision B, 15 February 1999.

2. ED27-00-099, Materials Science and Research Rack Number one International Standard Payload Rack Free-Free Transfer Function Test, ISS-DEV-00-054, NASA/MSFC/ED27, 26 July 2000.

3. Clough, R. W., and Penzien, J., Dynamics of Structures, McGraw Hill Inc., New York, NY, 1993. 